\title{
EXISTENCE OF MINIMAL SURFACES WITH A SIMPLE POLE AT INFINITY AND CONDITION OF TRANSVERSALITY ON THE SURFACE OF A CYLINDER
}

\author{
BY \\ YU WHY CHEN
}

Introduction. Let $\bar{C}$ be an infinite cylinder in the $x, y, z$ space, which is generated by a smooth curve $C$ in the $x, y$ plane through parallel displacement along the $z$-axis. Consider a steady, irrotational flow around $\bar{C}$, which is independent of $z$, with the velocity potential $\varphi(x, y)$. Instead of the general pressure-density law of the flow, we shall consider as an approximation that the pressure is linearly dependent on the reciprocal density $\left({ }^{1}\right)$. The differential equation for the velocity potential $\varphi(x, y)$ of a subsonic flow becomes then the differential equation of a minimal surface $\left({ }^{2}\right)$ :

$$
\left[1+\left(\frac{\partial \varphi}{\partial y}\right)^{2}\right] \frac{\partial^{2} \varphi}{\partial x^{2}}-2 \frac{\partial \varphi}{\partial x} \frac{\partial \varphi}{\partial y} \frac{\partial^{2} \varphi}{\partial x \partial y}+\left[1+\left(\frac{\partial \varphi}{\partial x}\right)^{2}\right] \frac{\partial^{2} \varphi}{\partial y^{2}}=0 .
$$

Our main interest is to investigate the existence of a steady, irrotational, subsonic flow without circulation, in the exterior of the curve $C$, given the velocity at infinity and the boundary condition $\partial \varphi / \partial n=0$ on $C$.

By using the symbol $z$ instead of $\varphi$ and upon choosing a proper $x, y$ coordinate system we can formulate the problem as follows:

Problem I. Given a simple closed smooth curve $C$ in the $x, y$ plane to find a minimal surface in the $x, y, z$ space, which is defined in the exterior of $C$ and represented by $z=z(x, y)$ with $\partial z / \partial x \rightarrow \beta, \partial z / \partial y \rightarrow 0$ at infinity and the condition of transversality on the cylinder $\bar{C}$.

This problem is of importance both for practical engineering and for the theory of compressible fluids. A study of the velocity and pressure distribution around a cylindrical body has been made by von Kármán and Tsien $\left({ }^{3}\right)$,

Presented to the Society, December 29, 1946; received by the editors January 22, 1948.

(1) This is the method suggested by A. Chaplygin to treat the subsonic flow of compressible fluid. Using this assumption Chaplygin obtained for the velocity potential and the stream-function two linear partial differential equations with flow speed and flow direction as independent variables. See for reference: von Kármán, The engineer grapples with the nonlinear problems, Bull. Amer. Math. Soc. vol. 46 (1940) p. 671.

(2) See for instance H. Bateman, Note on differential equation which occurs in the two dimensional motion of a compressible fluid and the associated variational problem, Proc. Roy. Soc. London Ser. A. vol. 125 (1929) p. 598. I want to thank C. C. Lin for first calling to my attention this relationship between minimal surfaces and theory of compressible fluid.

(3) H. S. Tsien, Two dimensional subsonic flow of compressible fluids, Journal of the Aeronautical Sciences vol. 6 (1939) pp. 399--407. 
using an approximate solution of the problem. Other authors have extended the considerations to flow with circulations $\left({ }^{4}\right)$. However, with Chaplygin's method one encounters difficulties in formulating the boundary condition.

From the purely mathematical point of view the problem is also of great interest. The question in general of the existence of a minimal surface which behaves at infinity like a plane with a given normal direction and which cuts a given cylinder orthogonally is in the line of the modern development of Plateau's problem. It is the method of the variational calculus, developed in the treatment of the Plateau problem $\left({ }^{5}\right)$, which is used here in this paper.

We shall formulate the problem in the parametric form. We shall try to find the minimal surface represented by three functions $x(u, v), y(u, v)$ and $z(u, v)$ harmonic in the interior of the unit circle $R: u^{2}+v^{2}<1$ with

$$
x_{u}^{2}+y_{u}^{2}+z_{u}^{2}=x_{v}^{2}+y_{v}^{2}+z_{v}^{2}, \quad x_{u} x_{v}+y_{u} y_{v}+z_{u} z_{v}=0 .
$$

Let $(u, v)=(0,0)$ correspond to the point at infinity in the $x, y$ plane. From the condition at infinity we know the behavior of the three harmonic functions $x(u, v), y(u, v)$ and $z(u, v)$ at $(u, v)=(0,0)$. The boundary condition gives $\partial z / \partial r=0$ at $r=1$, where $r, \theta$ are polar coordinates with $r e^{i \theta}=u+i v$. Upon proper choice of the $u, v$ system the three functions will be represented as follows

$$
\begin{aligned}
& x(u, v)=-a\left(1+\beta^{2}\right)^{-1 / 2}(1 / r-r) \cos \theta+X(u, v), \\
& y(u, v)=a(1 / r-r) \sin \theta+Y(u, v), \\
& z(u, v)=-\beta a\left(1+\beta^{2}\right)^{-1 / 2}(1 / r+r) \cos \theta+b .
\end{aligned}
$$

We now state the problem in the parametric form as Problem II.

Problem II. To find two functions, $X(u, v)$ and $Y(u, v)$ harmonic in the interior of the unit circle, which map the positive oriented unit circle into the negative oriented Jordan curve $C$ in a monotonic and continuous way, furthermore to find a constant $a \neq 0$ such that the three functions $x(u, v), y(u, v)$ and $z(u, v)$ in (1.9) represent a minimal surface. $b$ is an arbitrary constant.

The two functions $X(u, v)$ and $Y(u, v)$ will be obtained as a solution of the following

Minimum problem: Among all the function pairs $f(u, v), g(u, v)$ which are

(4) L. Bers, On a method of constructing two dimensional subsonic flow around closed profiles, NACA TN No. 969 (1945); On the circulatory subsonic flow of a compressible fluid past a circular cylinder, NACA TN No. 970 (1945): S. Bergman, On two dimensional flows of compressible fluids, NACA TN No. 972 (1945); C. C. Lin, On the extension of the von Karman-Tsien method to two dimensional subsonic flows with circulation around closed profiles, Quarterly of Applied Mathematics vol. 4 (1946).

(5) J. Douglas, The problem of Plateau, Bull. Amer. Math. Soc. vol. 39 (1933) p. 227 ff.; T. Rad6, The problem of Plateau, Ergebnisse der Mathematik, Berlin, 1933; R. Courant, Plateau's problem and Dirichlet's principle, Ann. of Math. vol. 38 (1937) pp. 679 ff.; R. Courant and D. Hilbert. Methoden der Mathematischen Physik, vol. 2, 1937, chap. 7. 
continuous in $\bar{R}: u^{2}+v^{2} \leqq 1$, piecewise differentiable in $R: u^{2}+v^{2}<1$, and map the positive oriented unit circle into the negative oriented Jordan curve $C$ in a monotonic and continuous way, find the pair $X(u, v), Y(u, v)$ which makes

$$
I(f, g)=\iint_{R}\left(f_{u}^{2}+f_{v}^{2}+g_{u}^{2}+g_{v}^{2}\right) d u d v-\frac{1}{\pi}\left(\int_{r=1} f d v+\left(1+\beta^{2}\right)^{1 / 2} g d u\right)^{2}
$$

a minimum.

Once the existence of the solution of the minimum problem has been proved, we put it into (1.9) and set

$$
a=-\frac{1+\beta^{2}}{2 \pi} \int_{r=1}\left(X /\left(1+\beta^{2}\right)^{1 / 2} d v+Y d u\right) ;
$$

then the three functions $x(u, v), y(u, v)$ and $z(u, v)$ represent a minimal surface, which solves problem II.

For $\beta=0$, the surface normal at infinity is parallel to the $z$-axis, any plane $z=$ const. is obviously a solution of our problem. Indeed the solution in (1.9) obtained in this case is the 1-1 conformal mapping of the unit circle $u^{2}+v^{2} \leqq 1$ into the closed domain in the exterior of $C$ and bounded by $C$. Consider any sequence of constants $\beta_{i}$ with $\beta_{i} \rightarrow \beta^{*}$ as $i \rightarrow \infty$ and let $x_{i}, y_{i}, z_{i}$ be a solution of problem II with $\beta=\beta_{i}$; then this sequence of solutions will converge to a solution of problem II in which $\beta=\beta^{*}$. This will be proved at the end of the present paper. Note that $\beta^{2}=M^{2} /\left(1-M^{2}\right)$, where $M$ is the Mach number of the flow at infinity. We may mention in this connection that extensive calculations have been carried out by different authors for subsonic flow around a circular cylinder $\left(^{6}\right)$ under the assumption that the velocity potential can be developed as a power series in $M^{2}$.

Any solution of problem I is a solution of problem II. The question whether any solution of problem II is a solution of problem I remains to be settled. We hope to come back to the investigation of this question in a later paper. A solution of problem II is a solution of problem I with a weak form of the condition of transversality only if a solution of it satisfies the conditions (i) the $x(u, v), y(u, v)$ map the unit circle topologically into $C$, (ii) the Jacobian $\partial(x, y) / \partial(u, v) \neq 0$ in $R$.

1. The problem and its formulation in the parametric form. Let the minimal surface be represented by three functions $x(u, v), y(u, v)$ and $z(u, v)$ which are the real parts of three analytic functions

$$
x(u, v)=R \varphi_{1}(w), \quad y(u, v)=R \varphi_{2}(w), \quad z(u, v)=R \varphi_{3}(w)
$$

in the complex variable $w=u+i v$, defined in the interior $R$ of the unit circle: $|w|<1$, with the identity

$$
\left(d \varphi_{1}\right)^{2}+\left(d \varphi_{2}\right)^{2}+\left(d \varphi_{3}\right)^{2} \equiv 0 .
$$

(6) See von Kármán, loc. cit. footnote 1 pp. 668-669. 
Let the origin $w=0$ correspond to the point at infinity of the $x, y$ plane. Then the analytic functions $\varphi_{1}(w), \varphi_{2}(w)$ and $\varphi_{3}(w)$, or at least two of them, will have a singularity at the origin. Since

$$
\partial z / \partial x \rightarrow \beta, \quad \partial z / \partial y \rightarrow 0
$$

as $(x, y)$ tends to infinity, the surface normal has at $w=0$ the limit direction $-\beta: 0: 1$. Furthermore the surface consists of one sheet extended to infinity. It follows $\left({ }^{7}\right)$ that the singularity must be a simple pole. If we denote for a moment the directions $-\beta: 0: 1$ as the direction of the $z^{\prime}$-axis of an orthogonal coordinate system $x^{\prime}, y^{\prime}, z^{\prime}$, then our minimal surface expressed in this system will have the following local development at $w=0$ :

$$
\begin{aligned}
x^{\prime}(u, v) & =R \psi_{1}(w), \quad y^{\prime}(u, v)=R \psi_{2}(w), \quad z^{\prime}(u, v)=R \psi_{3}(w), \\
d \psi_{1} & =a w^{-2}+\cdots, \quad d \psi_{2}=-i a w^{-2}+\cdots, \quad d \psi_{3}=c w^{m}+\cdots,
\end{aligned}
$$

where $m \geqq-1$, and $a$ is a complex number different from zero. By a suitable rotation of the $u, v$ plane we can make the coefficient real, which we shall assume to be true henceforth. Returning to the fixed $x, y, z$ coordinate system, which is connected with the $x^{\prime}, y^{\prime}, z^{\prime}$ system by the transformation

$$
\begin{aligned}
& x=\left(1+\beta^{2}\right)^{-1 / 2} x^{\prime}-\beta\left(1+\beta^{2}\right)^{-1 / 2} z^{\prime}, \\
& z=\beta\left(1+\beta^{2}\right)^{-1 / 2} x^{\prime}+\left(1+\beta^{2}\right)^{-1 / 2} z^{\prime}, \\
& y=y^{\prime},
\end{aligned}
$$

we obtain the local development of $d \varphi_{i}$

$$
\begin{aligned}
& d \varphi_{1}=a /\left(1+\beta^{2}\right)^{1 / 2} w^{-2}-\beta a /\left(1+\beta^{2}\right)^{1 / 2} w^{m}+\cdots, \\
& d \varphi_{2}=-i a w^{-2}+\cdots, \\
& d \varphi_{3}=a \beta\left(1+\beta^{2}\right)^{-1 / 2} w^{-2}+c\left(1+\beta^{2}\right)^{-1 / 2} w^{m}+\cdots
\end{aligned}
$$

at $w=0$. This development expresses the behavior of the surface at infinity in the parametric form. If $m=-1$, then in (1.4) some of the functions will have a logarithmic term, thus becoming multi-valued. The corresponding differentials will have nonzero periods. This case is important for study of the subsonic flow with circulation. However, in the present paper we shall not investigate this case, but assume $m>-1$. Then we derive from (1.4) the representation of $\varphi_{i}(w)$ by

$$
\begin{aligned}
& \varphi_{1}(w)=-a\left(1+\beta^{2}\right)^{-1 / 2} w^{-1}+\phi_{1}(w), \\
& \varphi_{2}(w)=i a w^{-1}+\phi_{2}(w), \\
& \varphi_{3}(w)=-\beta a\left(1+\beta^{2}\right)^{-1 / 2} w^{-1}+\phi_{3}(w)
\end{aligned}
$$

and their real parts:

( $\left.{ }^{7}\right)$ See for reference a paper of the present author: Branchpoints, poles and planar points of minimal surfaces in $R^{3}$, Ann. of Math. vol. 49 (1948). 


$$
\begin{aligned}
& x(u, v)=-a\left(1+\beta^{2}\right)^{-1 / 2}(1 / r-r) \cos \theta+X(u, v), \\
& y(u, v)=a(1 / r-r) \sin \theta+Y(u, v), \\
& z(u, v)=-\beta a\left(1+\beta^{2}\right)^{-1 / 2}(1 / r+r) \cos \theta+Z(u, v),
\end{aligned}
$$

where in (1.5) $\phi_{i}(w)$ are regular analytic functions in $|w|<1$, and $a(1+\beta)^{-1 / 2}(r \cos \theta)+X(u, v)$ is the real part of $\phi_{1}(w)$, and so on. The three functions $X(u, v), Y(u, v)$ and $Z(u, v)$ are harmonic functions in $|w|<1$ without singularities. The way we represent $x, y, z$ in (1.6) by writing the real part of $\phi_{1}(w)$ as the sum of $a\left(1+\beta^{2}\right)^{-1 / 2}(r \cos \theta)$ and $X(u, v)$, and so on, will make it easier to formulate the boundary conditions.

In the parametric representation of the surface the circle $r=1$ should correspond to the boundary of the minimal surface. The condition of transversality means that surface normals should exist on the boundary and lie in the tangent plane of the cylinder $\bar{C}$. Hence we have for $r=1$ the condition

$$
\left(y_{r} z_{\theta}-z_{r} y_{\theta}\right):\left(z_{r} x_{\theta}-x_{r} z_{\theta}\right)=x_{\theta}: y_{\theta}
$$

when the first two components of the surface normal $\left(y_{r} z_{\theta}-z_{r} y_{\theta}\right),\left(z_{r} x_{\theta}-x_{r} z_{\theta}\right)$ are not both zero, and in the latter case we have the third component $\left(x_{r} y_{\theta}-y_{r} x_{\theta}\right) \neq 0$. This condition, together with the identity (1.2) which is satisfied by any minimal surface, leads to the boundary condition for the function $z(u, v)$ :

$$
\partial z / \partial r=0,
$$$$
\text { for } r=1 \text {. }
$$

But the function $z(u, v)$ is a harmonic function. The condition (1.8) determines $z(u, v)$ therefore up to a constant. By writing $z(u, v)$ in the form as represented in (1.6) we see that the unknown function $Z(u, v)$ is a constant. By a translation of the coordinate system $x, y, z$ along the $z$-axis we can put $Z(u, v) \equiv 0$. Thus our minimal surface is represented by

$$
\begin{aligned}
& x(u, v)=-a\left(1+\beta^{2}\right)^{-1 / 2}(1 / r-r) \cos \theta+X(u, v), \\
& y(u, v)=a(1 / r-r) \sin \theta+Y(u, v), \\
& z(u, v)=-\beta a\left(1+\beta^{2}\right)^{-1 / 2}(1 / r+r) \cos \theta,
\end{aligned}
$$

with two unknown functions $X(u, v)$ and $Y(u, v)$ and an unknown constant $a \neq 0$. For these unknowns we pose the problem II which has been formulated in the introduction of the present paper.

2. Problem II as a minimum problem in the calculus of variations. In the modern treatment of the Plateau problem minimal surfaces are characterized as those among all surfaces with a given boundary which give a minimum or stationary value to the Dirichlet integral $\left({ }^{8}\right)$. If we set up the Dirichlet integral for the three functions in (1.9), then, due to the existence of a pole,

(8) Note that problem I is different from a Plateau problem, but that its reduction to problem II suggests a similar treatment. 
this integral becomes infinite. To overcome this difficulty we follow a device used by $\mathrm{H}$. Weyl $\left({ }^{9}\right)$ when he established the existence of harmonic functions with prescribed singularities on a closed surface. In the interior of the unit circle, we cut a small circle with radius $\rho(0<\rho<1)$ around the singularity at the origin and denote the region $0 \leqq r<\rho$ by $R_{\rho}$. The three functions $x(u, v), y(u, v)$ and $z(u, v)$ will be replaced by another three functions $x^{\prime}(u, v)$ $y^{\prime}(u, v)$ and $z^{\prime}(u, v)$ in that part of the Dirichlet integral which extends over the region $R_{p}$. These new functions are defined in $R_{p}$ as follows:

$$
\begin{aligned}
& x^{\prime}(u, v)=x(u, v)-t^{1}(u, v), \\
& y^{\prime}(u, v)=y(u, v)-t^{2}(u, v), \\
& z^{\prime}(u, v)=z(u, v)-t^{3}(u, v),
\end{aligned}
$$

with the functions $t^{i}(u, v), i=1,2,3$, possessing the properties: (1) $t^{1}, t^{2}, t^{3}$ have the same singularity as the $x, y, z$ have respectively so that $x^{\prime}, y^{\prime}, z^{\prime}$ are regular in $R_{\rho}$, (2) the normal derivatives $\partial t^{i} / \partial r=0, i=1,2,3$, on the circle with radius $\rho$. This determines the functions $t^{i}$ uniquely, if we require that they are harmonic functions:

$$
\begin{aligned}
t^{1} & =-a\left(1+\beta^{2}\right)^{-1 / 2}\left(1 / r \cos \theta+r / \rho^{2} \cos \theta\right), \\
t^{2} & =a\left(1 / r \sin \theta+r / \rho^{2} \sin \theta\right), \\
t^{3} & =-a \beta\left(1+\beta^{2}\right)^{-1 / 2}\left(1 / r \cos \theta+r / \rho^{2} \cos \theta\right) .
\end{aligned}
$$

We form the Dirichlet integral $D_{R_{\rho}}\left(x^{\prime}, y^{\prime}, z^{\prime}\right)$ and the Dirichlet integral of the original functions $x, y, z: D_{R-R_{\rho}}(x, y, z)$ over the ring region $o^{2}<u^{2}+v^{2}<1$ which we denote by $R-R_{\rho}$. Let the sum of these two Dirichlet integrals be denoted by $I_{\rho}$ :

$$
I_{\rho}=D_{R-R_{\rho}}(x, y, z)+D_{R_{\rho}}\left(x^{\prime}, y^{\prime}, z^{\prime}\right) .
$$

By putting in (2.2) the expressions of $x, y, z$ from (1.9) and the expressions of $x^{\prime}, y^{\prime}, z^{\prime}$ from (2.1) one finds through some computations the result:

$$
\begin{aligned}
I_{\rho}= & D_{R}(X, Y)+4 a \int_{r=1}\left(\left(1+\beta^{2}\right)^{-1 / 2} X d v+Y d u\right) \\
& +4 \pi a^{2} / \rho^{2}+4 \pi a^{2} /\left(1+\beta^{2}\right)
\end{aligned}
$$

where $D_{R}(X, Y)$ denotes the Dirichlet integral of the two unknown functions $X(u, v)$ and $Y(u, v)$ over $R$, and $a$ is the same constant as in (1.9). The value of $I_{\rho}$ depends on the functions $X(u, v), Y(u, v)$ and the constants $a, \rho$ and $\beta$.

A pair of continuous functions $X(u, v), Y(u, v)$ in $\bar{R}: u^{2}+v^{2} \leqq 1$ is called an admissible pair, if they are piecewise differentiable in $R$ and map the positive oriented circle $u^{2}+v^{2}=1$ into the negative oriented curve $C$ in a monotonic and continuous way. For each admissible pair $X(u, v), Y(u, v)$ we determine

(9) H. Weyl, Die Idee der Riemannschen Flaeche, Leipzig. 
the constant $a$ in such a way that the expression $I-4 \pi a^{2} / \rho^{2}$ attains its minimum. This gives immediately the value of $a\left({ }^{10}\right)$

$$
a=-\frac{1+\beta^{2}}{2 \pi} \int_{r=1}\left(\left(1+\beta^{2}\right)^{-1 / 2} X d v+Y d u\right)
$$

in terms of a line integral of the functions $X, Y$ over the circle $r=1$. For all admissible pairs the right side of (2.4) is uniformly bounded, the same is therefore true for the values of $a$. Let $N$ be an upper bound of $a$. For each $a$ obtained in (2.4) we choose $\rho^{2}=a^{2} /\left(N^{2}+1\right)<1$. The quotient $a^{2} / \rho^{2}$ becomes now a fixed constant $\left.{ }^{11}\right)$. Consequently, by putting (2.4), the value of $a$, into (2.3) and choosing $\rho$ as mentioned, any admissible pair $X, Y$ which minimizes the expression $I_{\rho}$ will also minimize the expression $I_{\rho}-4 \pi a^{2} / \rho^{2}$ and conversely. Let the last expression be denoted by $I(X, Y)$. It is independent of $\rho$ and by (2.3) and (2.4) we have

$$
I(X, Y)=D_{R}(X, Y)-\frac{1}{\pi}\left(\int_{r=1} X d v-Y\left(1+\beta^{2}\right)^{1 / 2} d u\right)^{2} .
$$

The existence of an admissible pair of functions which minimize the integral $I(X, Y)$ will be proved in the next section.

Let us denote the line in tegral on the right side of $(2.5)$ by $J(X, Y)$, so that (2.5) can be written as

$$
I(X, Y)=D_{R}(X, Y)-(1 / \pi) J^{2}(X, Y) .
$$

The integral $I(X, Y)$ is not invariant under all orthogonal transformations in the $X, Y$ plane, because the line integral $J(X, Y)$ is not. Furthermore $J(X, Y)$ will change its value by a conformal transformation

$$
w=e^{i \eta}\left(w^{\prime}-\lambda e^{i \mu}\right) /\left(1-\lambda e^{-i \mu} w^{\prime}\right)
$$

of the unit circle $|w| \leqq 1$ into itself, in the following way. First we have by the mean value theorem of harmonic functions

$$
\begin{aligned}
J(X, Y) & =\iint_{R}\left(X_{u}-Y_{v}\left(1+\beta^{2}\right)^{1 / 2}\right) d u d v \\
& =\pi\left(X_{u}-Y_{v}\left(1+\beta^{2}\right)^{1 / 2}\right)_{w=0} .
\end{aligned}
$$

From the transformation (2.6) we obtain another pair of harmonic functions $X^{\prime}\left(u^{\prime}, v^{\prime}\right)$ and $Y^{\prime}\left(u^{\prime}, v^{\prime}\right)$ in the parameters $u^{\prime}$ and $v^{\prime}\left(w^{\prime}=u^{\prime}+i v^{\prime}\right)$ by setting

$$
X^{\prime}\left(u^{\prime}, v^{\prime}\right)=X(u, v), \quad Y^{\prime}\left(u^{\prime}, v^{\prime}\right)=Y(u, v) .
$$

(10) That we should put $a$ equal to this value can also be seen as follows: If the functions in (1.9) represent a minimal surface, then by putting these functions into the identity (1.2) and comparing the coefficients of the power $1 / w^{2}$ one finds the value of $a$ as in (2.4).

(11) When $a=0$, we put $\rho=0$. This case can be excluded from the consideration, as we shall see presently. 
We form the line integral $J\left(X^{\prime}, Y^{\prime}\right)$ in the parameter $u^{\prime} v^{\prime}$. It is seen immediately due to (2.7) and the transformation rules

$$
\begin{aligned}
d w / d w^{\prime} & =e^{i \eta}\left(1-\lambda^{2}\right) /\left(1-\lambda e^{-i \mu} w^{\prime}\right)^{2}, \\
X_{u^{\prime}}^{\prime}-i X_{v^{\prime}}^{\prime} & =\left(X_{u}-i X_{v}\right) d w / d w^{\prime}, \\
Y_{u^{\prime}}^{\prime}-i Y_{v^{\prime}}^{\prime} & =\left(Y_{u}-i Y_{v}\right) d w / d w^{\prime}
\end{aligned}
$$

that

$$
\begin{aligned}
J\left(X^{\prime}, Y^{\prime}\right)= & \left(X_{u^{\prime}}^{\prime}-Y_{v^{\prime}}^{\prime}\left(1+\beta^{2}\right)^{1 / 2}\right)_{w^{\prime}=0} \\
= & \pi\left(1-\lambda^{2}\right)\left[\left(X_{u}-Y_{v}\left(1+\beta^{2}\right)^{1 / 2}\right) \cos \eta\right. \\
& \left.+\left(X_{v}+Y_{u}\left(1+\beta^{2}\right)^{1 / 2}\right) \sin \eta\right]_{w=-\lambda e^{i(\mu+\eta)}}
\end{aligned}
$$

By suitable choice of $\eta$ the above expression can attain the value

(2.8) $\pi\left(1-\lambda^{2}\right)\left[\left(X_{u}-Y_{v}\left(1+\beta^{2}\right)^{1 / 2}\right)^{2}+\left(X_{v}+Y_{u}\left(1+\beta^{2}\right)^{1 / 2}\right)^{2}\right]_{v=-\lambda e^{i \theta}}^{1 / 2}$

with $\theta=\mu+\eta$.

LEMMA 1. There is no admissible pair of harmonic functions $X(u, v), Y(u, v)$ such that, for all conformal transformations $w \rightarrow w^{\prime}$ of the unit circle into itself, the line integral $J(X, Y)$ will be zero.

Proof. For such a pair we would have

$$
X_{u}-Y_{v}\left(1+\beta^{2}\right)^{1 / 2}=0, \quad X_{v}+Y_{u}\left(1+\beta^{2}\right)^{1 / 2}=0
$$

at any point in the interior of the unit circle. This shows that $X-i\left(1+\beta^{2}\right)^{1 / 2} Y$ is an analytic function of the complex variable $w$. As the parameter $(u, v)$ runs along the unit circle in the positive sense, the point $(X, Y)$ will describe the curve $C$, and hence the point $\left(X,\left(1+\beta^{2}\right)^{1 / 2} \cdot Y\right)$ describes a curve $C^{\prime}$ in the $(X, Y)$-plane in the negative sense in a monotonic and continuous way. Suppose the origin of the $X, Y$ plane lies in the interior of $C^{\prime}$, then $(1 / 2 \pi i) \int_{r=1} d \log \left(X-i\left(1+\beta^{2}\right)^{1 / 2} Y\right)=-1$. But this contradicts the fact that the analytic function $X-i\left(1+\beta^{2}\right)^{1 / 2} Y$ is bounded in $R$ and hence has no pole there.

Lemma 2. Suppose $X(u, v), Y(u, v)$ is a pair of harmonic functions which solves the minimum problem $I(X, Y)=\min$. Then $J(X, Y) \neq 0$.

Proof. If $J(X, Y)=0$, then according to Lemma 1 there is a conformal transformation of $R$ into itself, which transforms the $X, Y$ into another pair $X^{\prime}, Y^{\prime}$ of admissible harmonic functions, with $J\left(X^{\prime}, Y^{\prime}\right) \neq 0$. Since the Dirichlet integrals for the two pairs of functions have the same value, it follows that $I(X, Y)$ is greater than $I\left(X^{\prime}, Y^{\prime}\right)$, which is impossible. The fact that $J(X, Y) \neq 0$ assures the nonvanishing of the leading terms on the right side of (1.9). 
3. Existence of a solution of the minimum problem. We shall use the direct method of the calculus of variations to establish the existence of a solution of the minimum problem:

$$
I(X, Y)=\min \text {. }
$$

among all the admissible pairs of functions $X(u, v), Y(u, v)$ defined in the last section. Our integral is a sum of two integrals: the Dirichlet integral and the line integral $J$. The latter depends only upon the boundary value and is uniformly bounded from above and below for all admissible functions. Since the Dirichlet integral is never negative, it follows that the integral $I(X, Y)$ is uniformly bounded from below. Therefore the set of values assumed by $I(X, Y)$ for all admissible pairs has a lower limit. We denote this limit by $d$. There exists a minimizing sequence of admissible pairs $X_{i}, Y_{i}, i=1,2,3$, with

$$
I\left(X_{i}, Y_{i}\right) \rightarrow d, \quad i \rightarrow \infty .
$$

We observe that the Dirichlet integral $D\left(X_{i}, Y_{i}\right)$ for any minimizing sequence is bounded from above. This follows from (3.1) and the fact that $J\left(X_{i}, Y_{i}\right)$ is bounded. The boundedness of the Dirichlet integral enables us to use Courant's lemma $\left({ }^{12}\right)$ which we state in the special form needed here:

Courant's Lemma. For a set of admissible function pairs in $\bar{R}$, $f_{i}(u, v), g_{i}(u, v)$, whose Dirichlet integrals are uniformly bounded by a constant $M$ :

$$
D_{R}\left(f_{i}, g_{i}\right)<M,
$$

the following is true: to each $\delta, 1>\delta>0$, and at each point $Q$ in $\bar{R}$ there is a $\rho$ with $\delta<\rho<\delta^{1 / 2}$ such that the circular arc with $Q$ as center, $\rho$ as radius, lying in $\bar{R}$, will be mapped by $f_{i}, g_{i}$ into an arc of length not greater than $(2 \pi \epsilon(\delta))^{1 / 2}$, $\epsilon(\delta)=2 M / \log (1 / \delta), \epsilon(\delta) \rightarrow 0$, as $\delta \rightarrow 0$.

The curve $C$ is a closed Jordan curve, it has therefore the property: there exists for each $\tau>0$ a $\sigma(\tau)>0$, with $\sigma(\tau) \rightarrow 0$ for $\tau \rightarrow 0$, so that for any pair of point $A, B$ on $C$ whose distance $\|A B\|$ from each other is not greater than $\tau$, one of the two arcs $A B$ has a diameter not exceeding $\sigma(\tau)$.

We now apply Courant's Lemma to our minimizing sequence $X_{i}(u, v)$, $Y_{i}(u, v)$ in order to establish the equi-continuity of the set of functions $X_{i}(\cos \theta, \sin \theta), Y_{i}(\cos \theta, \sin \theta)$ in the independent variable $\theta, 0 \leqq \theta \leqq 2 \pi$, $i=1,2,3 \cdots$. Let the mapping of the unit circle $u^{2}+v^{2}=1$ into $C$ by the pair of functions $X_{i}, Y_{i}$ be denoted by $T_{i}$. We consider for a fixed $i$ the images $T_{i}(P), T_{i}(Q)$ of any two points $P$ and $Q$ on the unit circle, with the distance $\|P Q\|$ of $P$ and $Q$ from each other being less than $2 \delta, 1>\delta>0$. From Courant's Lemma follows: either

(12) R. Courant and D. Hilbert, Methoden der Mathematischen Physik, vol. 2, pp. 541-544. 


$$
\left\|T_{i}(P) T_{i}(Q)\right\| \leqq \sigma(\tau), \quad \tau=(2 \pi \epsilon(\delta))^{1 / 2}, \quad \text { for }\|P Q\|<2 \delta,
$$

or one can find on the unit circle an $\operatorname{arc} \gamma$ which contains $P$ and $Q$ in the interior and which has the two points $P^{\prime}$ and $Q^{\prime}$ as its end points with $\left\|P^{\prime} Q^{\prime}\right\|<2 \delta^{1 / 2}$; the complimentary arc of $\gamma$ will be mapped by $T_{i}$ into an arc (on $C$ ) whose diameter is less than $\sigma(\tau), \tau=(2 \pi \epsilon(\delta))^{1 / 2}$. If we can show that the last of the two above possibilities never occurs, that means (3.2) is true for any $i$, then we have the equi-continuity of the set functions $X_{i}(\cos \theta, \sin \theta)$, $Y_{i}(\cos \theta, \sin \theta), i=1,2,3, \cdots$, since the $\delta$ and $\sigma(\tau)$ were chosen independently of $i$.

We first prove the following lemma.

Lемма 3. There is always a minimizing sequence with the line integrals $J\left(X_{i}, Y_{i}\right)$ uniformly bounded away from zero:

$$
J\left(X_{i}, Y_{i}\right)>k>0
$$

where $k$ is a constant independent of $i$.

Proof. We can assume that in a minimizing sequence all the functions are harmonic functions, since we can replace each pair $X_{i}, Y_{i}$ by a pair of harmonic functions with the same boundary value, where the line integral is unchanged and the Dirichlet integral does not increase. For each pair of harmonic functions in the minimizing sequence we can further assume that the line integral $J \neq 0$; this follows from Lemma 1. Suppose now for this minimizing sequence the line integrals are not bounded away from zero, then there must exist a subsequence with $J\left(X_{i}, Y_{i}\right) \rightarrow 0$. Of course this subsequence is again a minimizing sequence. We transform each pair $X_{i}, Y_{i}$ into a new pair of harmonic functions $X_{i}^{\prime}, Y_{i}^{\prime}$ by performing a conformal transformation $M_{i}:(u, v) \rightarrow\left(u^{\prime}, v^{\prime}\right)$ of the unit circle $u^{2}+v^{2} \leqq 1$ into itself and assigning $X^{\prime}\left(u^{\prime}, v^{\prime}\right)=X(u, v), Y^{\prime}\left(u^{\prime}, v^{\prime}\right)=Y(u, v)$. We get a new sequence of admissible functions $X_{i}^{\prime}, Y_{i}^{\prime}, i=1,2,3 \cdots$. I say this new sequence has also the property $J\left(X_{i}^{\prime}, Y_{i}^{\prime}\right) \rightarrow 0$, as $i \rightarrow \infty$, no matter how the conformal transformations $M_{i}$ are chosen. For, $D\left(X_{i}, Y_{i}\right)=D\left(X_{i}^{\prime}, Y_{i}^{\prime}\right)$ and

$$
\begin{aligned}
\lim _{i \rightarrow \infty} I\left(X_{i}, Y_{i}\right) & =\lim _{i \rightarrow \infty}\left[D\left(X_{i}, Y_{i}\right)-(1 / \pi) J^{2}\left(X_{i}, Y_{i}\right)\right]=d \\
& =\lim _{i \rightarrow \infty} D\left(X_{i}, Y_{i}\right)=\lim _{i \rightarrow \infty} D\left(X_{i}^{\prime}, Y_{i}^{\prime}\right),
\end{aligned}
$$

since $J\left(X_{i}, Y_{i}\right) \rightarrow 0$. If now $J\left(X_{i}^{\prime}, Y_{i}^{\prime}\right)$ does not converge to zero, as $i \rightarrow \infty$, then due to (3.4) we would have a pair of functions $X_{N}^{\prime}, Y_{N}^{\prime}$ for which $I\left(X_{N}^{\prime}, Y_{N}^{\prime}\right)<d$. This is impossible. Hence $J\left(X_{i}^{\prime}, Y_{i}^{\prime}\right) \rightarrow 0$, as $i \rightarrow \infty$, and $\lim _{i \rightarrow \infty} I\left(X_{i}^{\prime}, Y_{i}^{\prime}\right)=d$. Therefore the sequence $X_{i}^{\prime}, Y_{i}^{\prime}, i=1,2,3, \cdots$, is minimizing. Upon proper choice of the mappings $M_{i}$ the sequence $X_{i}^{\prime}, Y_{i}^{\prime}$ will satisfy a "three points condition" $\left({ }^{13}\right)$, from which the equi-continuity of

${ }^{(13)}$ See Courant loc. cit. footnote 12. 
$X_{i}^{\prime}(\cos \theta, \sin \theta), Y_{i}^{\prime}(\cos \theta, \sin \theta), i=1,2,3, \cdots$, in the independent variable $\theta, 0 \leqq \theta<2 \pi$, follows.

Since our minimizing sequence consists of harmonic functions, the equicontinuity of their boundary values will have as consequence the compactness of the sequence. Due to the lower semi-continuity of the Dirichlet integral and the continuity of the line integral $J$, it follows that the limit function-pair, being admissible, will be a solution of the minimum problem with the property that the line integral $J$ of this function pair is zero. This contradicts Lemma 2. Thus Lemma 3 is proved.

We proceed now with a minimizing sequence $X_{i}, Y_{i}$ which satisfies (3.3), and show the validity of (3.2) for all $i$ and sufficiently small $\delta$. The proof is indirect and based on the following estimate. Suppose there is one pair of functions $X^{*}, Y^{*}$ in our minimizing sequence and two points $P$ and $Q$ on the unit circle, $\|P Q\|<2 \delta$, that (3.2) does not hold. Then it follows from Courant's Lemma that the alternative of (3.2) is true. We split the line integral $J\left(X^{*}, Y^{*}\right)$ into two parts, one over the arc $\gamma$ and another over its complementary arc $\bar{\gamma}$. $\gamma$ has the angular measure less than 2 arc $\sin \delta^{1 / 2} / 2$, and $\bar{\gamma}$ is mapped by $X^{*}, Y^{*}$ into $C$ on an arc of diameter less than $\sigma(\tau) . X^{*}(\cos \theta, \sin \theta), Y^{*}(\cos \theta$, $\sin \theta)$ are bounded by a constant $L$, which depends only on $C$. We have first

$$
\int_{\gamma} X^{*} d v+Y^{*}\left(1+\beta^{2}\right)^{1 / 2} d u<2\left(1+\left(1+\beta^{2}\right)^{1 / 2}\right) \arcsin \delta^{1 / 2} / 2,
$$

and also the equation

$$
\begin{gathered}
\int_{\bar{\gamma}} X^{*} d v+Y^{*}\left(1+\beta^{2}\right)^{1 / 2} d u=\int\left[X^{*} \cos \theta-Y^{*}\left(1+\beta^{2}\right)^{1 / 2} \sin \theta\right] d \theta \\
=\int_{\gamma}\left[\left(X^{*}-x_{0}\right) \cos \theta-\left(1+\beta^{2}\right)^{1 / 2}\left(Y^{*}-y_{0}\right) \sin \theta\right] d \theta \\
+\int_{\bar{\gamma}}\left[x_{0} \cos \theta-\left(1+\beta^{2}\right)^{1 / 2} y_{0} \sin \theta\right] d \theta
\end{gathered}
$$

where $x_{0}, y_{0}$ are the coordinates of a point which lies on the image arc of $\bar{\gamma}$. Since $X^{*}-x_{0}$ and $Y^{*}-y_{0}$ in the integrand are smaller than $\sigma(\tau)$ in absolute value, we obtain the estimates

$$
\begin{aligned}
& \int_{\bar{\gamma}}\left(\left(X^{*}-x_{0}\right) \cos \theta-\left(1+\beta^{2}\right)^{1 / 2}\left(Y^{*}-y_{0}\right) \sin \theta\right) d \theta \\
& <2\left(1+\left(1+\beta^{2}\right)^{1 / 2}\right) \sigma(\tau), \\
& \int_{\bar{\gamma}}\left(x_{0} \cos \theta-\left(1+\beta^{2}\right)^{1 / 2} y_{0} \sin \theta\right) d \theta \\
& <L 2\left(1+\left(1+\beta^{2}\right)^{1 / 2}\right) \arcsin \delta^{1 / 2} / 2 .
\end{aligned}
$$


From (3.5) and (3.6) it follows that $J\left(X^{*}, Y^{*}\right)$ would be arbitrarily small, if $\delta$ is sufficiently small. This contradicts (3.3). Hence as soon as $\delta$ is sufficiently small, (3.2) holds for every function pair $X_{i}, Y_{i}$ in our minimizing sequence. Thus the equi-continuity of the sequence of functions $X_{i}(\cos \theta, \sin \theta)$, $Y_{i}(\cos \theta, \sin \theta)$ in the independent variable, $\theta, 0 \leqq \theta \leqq 2 \pi$, is proved. The compactness of the minimizing sequence follows as before. The limit function is a solution of our minimum problem. Due to (3.3) the line integral $J$ of this solution is not zero.

THEOREM 1. The problem

$$
I(X, Y)=D(X, Y)-(1 / \pi) J^{2}(X, Y)=\min .
$$

has at least one solution, which consists of two admissible harmonic functions with $J \neq 0$.

4. The first variation; solution of problem II. The solution $X(u, v)$, $Y(u, v)$ of the minimum problem, obtained in the last section by direct method of the calculus of variations, is now put into (1.9) and the constant $a$ there is set equal to $-\left(1+\beta^{2}\right)^{1 / 2} / 2 \pi \cdot J(X, Y) \neq 0$. We shall show that the three harmonic functions $x(u, v), y(u, v)$ and $z(u, v)$ in (1.9) represent a minimal surface. This means we are going to prove the identity

$$
p(w) \equiv\left(x_{u}-i x_{v}\right)^{2}+\left(y_{u}-i y_{v}\right)^{2}+\left(z_{u}-i z_{v}\right)^{2} \equiv 0
$$

in $R$. Since we have from (1.9)

$$
\begin{aligned}
x_{u}-i x_{v} & =a\left(1+\beta^{2}\right)^{-1 / 2}\left(1+1 / w^{2}\right)+\left(X_{u}-i X_{v}\right), \\
y_{u}-i y_{v} & =i a\left(1-1 / w^{2}\right)+\left(Y_{u}-i Y_{v}\right), \\
z_{u}-i z_{v} & =\beta a\left(1+\beta^{2}\right)^{-1 / 2}\left(-1+1 / w^{2}\right),
\end{aligned}
$$

the identity $p(w) w^{2} \equiv 0$ will give the following two differential equations:

$$
\begin{aligned}
\Im p(w) w^{2} \equiv & -2 r\left(X_{r} X_{\theta}+Y_{r} Y_{\theta}\right)-2 a\left(\left(1+\beta^{2}\right)^{-1 / 2} X_{v}+Y_{u}\right) \\
& +2 a\left(1+\beta^{2}\right)^{-1 / 2} r^{2}\left(X_{r} \sin \theta-r^{-1} X_{\theta} \cos \theta\right) \\
& +2 a r^{2}\left(Y_{r} \cos \theta+r^{-1} Y_{\theta} \sin \theta\right)
\end{aligned}
$$

and

$$
\begin{aligned}
\Re p(w) w^{2} \equiv & r^{2}\left(X_{r}^{2}-r^{-2} X_{\theta}^{2}\right)+r^{2}\left(Y_{r}^{2}-r^{-1} Y_{\theta}^{2}\right) \\
& +2 a\left(1\left(1+\beta^{2}\right)^{-1 / 2} X_{u}-Y_{v}+2 a\left(1+\beta^{2}\right)^{-1}\right) \\
& +2 a\left(1+\beta^{2}\right)^{-1 / 2} r^{2}\left(X_{r} \cos \theta+r^{-1} X_{\theta} \sin \theta\right) \\
& -2 a r^{2}\left(Y_{r} \sin \theta-r^{-1} Y_{\theta} \cos \theta\right) .
\end{aligned}
$$

Hence the requirement that $x(u, v), y(u, v), z(u, v)$ represent a minimal surface is equivalent to the requirement that the solution $X(u, v), Y(u, v)$ of our 
minimum problem satisfy the two differential equations (4.1) and (4.2) with $a=-\left(1+\beta^{2}\right)^{1 / 2} / 2 \pi \cdot J(X, Y)$. This will be proved by considering a suitable first variation $\left({ }^{14}\right)$ of the integral $I(X, Y)$ which has the value $d$.

Let $\lambda(r \cos \theta, r \sin \theta)$ be a continuously differentiable function in the unit circle $0 \leqq r \leqq 1,0 \leqq \theta \leqq 2 \pi$. We form for sufficiently small parameter $\epsilon$ a oneparameter family of transformations $M_{\epsilon}$ of the unit circle into itself:

$$
M_{\epsilon}\left\{\begin{array}{l}
r e^{i \theta} \rightarrow r e^{i \vartheta} \\
\theta=\vartheta+\epsilon \lambda(r \cos \vartheta, r \sin \vartheta) .
\end{array}\right.
$$

Since we have

$$
\partial \theta / \partial \vartheta=1+\epsilon \lambda_{\vartheta}, \quad \partial \theta / \partial r=\epsilon \lambda_{r},
$$

the Jacobian of the transformation is different from zero for $|\epsilon|<1 / \max \left|\lambda_{\vartheta}\right|$. Furthermore $\partial \theta / \partial \vartheta>0$. Thus $M_{\epsilon}$ is a one-to-one transformation, maps the circle $r=1$ monotonically into itself and is the identity transformation for $\epsilon=0$. These transformations are now used to define a one-parameter family of admissible pairs of functions $X^{*}(r \cos \theta, r \sin \theta ; \epsilon), Y^{*}(r \cos \theta, r \sin \theta ; \epsilon)$ for $0 \leqq r \leqq 1,0 \leqq \theta \leqq 2 \pi$. We stipulate

$$
\begin{aligned}
& X^{*}(r \cos \vartheta, r \sin \vartheta ; \epsilon)=X(r \cos (\vartheta+\epsilon \lambda), r \sin (\vartheta+\epsilon \lambda)), \\
& Y^{*}(r \cos \vartheta, r \sin \vartheta ; \epsilon)=Y(r \cos (\vartheta+\epsilon \lambda), r \sin (\vartheta+\epsilon \lambda)) .
\end{aligned}
$$

It is immediately seen that for $\epsilon=0$ the pair $X^{*}(u, v ; 0), Y^{*}(u, v ; 0)$ is the solution $X(u, v), Y(u, v)$ of our minimum problem.

In order to compute the first variation $\delta I(X, Y)$ we carry out the integral

$$
\begin{aligned}
I(\epsilon)= & \iint_{R}\left(X_{r}^{* 2}+r^{-2} X_{\vartheta}^{* 2}+Y_{r}^{* 2}+r^{2} Y_{\vartheta}^{* 2}\right) r d r d \vartheta \\
& -\frac{1}{\pi}\left(\int_{r=1}^{2 \pi}\left(X^{*} \cos \vartheta-\left(1+\beta^{2}\right)^{1 / 2} Y^{*} \sin \vartheta\right) d \vartheta\right)^{2}
\end{aligned}
$$

up to the first order of $\epsilon$. We have

$$
\begin{aligned}
\int_{0}^{2 \pi} X^{*} \cos \vartheta d \vartheta= & \int_{0}^{2 \pi} X \cos \theta d \theta \\
& +\epsilon \int_{0}^{2 \pi} X\left(\lambda \sin \theta+\lambda_{\theta} \cos \theta\right) d \theta+O\left(\epsilon^{2}\right) \\
\int_{0}^{2 \pi} Y^{*} \sin \vartheta d \vartheta= & \int_{0}^{2 \pi} Y \sin \theta d \theta \\
& +\epsilon \int_{0}^{2 \pi} Y\left(\lambda \cos \theta+\lambda_{\theta} \sin \theta\right) d \theta+O\left(\epsilon^{2}\right)
\end{aligned}
$$

${ }^{(14)}$ For the method of variation followed here, see R. Courant, loc. cit. footnote 12, p. 539. 
and the first variation of the Dirichlet integral equal to

$$
-2 \int_{r=1}^{2 \pi} r\left(X_{r} X_{\theta}+Y_{r} Y_{\theta}\right) d \theta
$$

Summing up we obtain the result:

$$
\begin{aligned}
\delta I(X, Y)= & -2 \int_{0}^{2 \pi} \lambda r\left(X_{r} X_{\theta}+Y_{r} Y_{\theta}\right) d \theta \\
& -\frac{4 a}{\left(1+\beta^{2}\right)^{1 / 2}} \int_{r=1}^{2 \pi}\left[X(\lambda \cos \theta)_{\theta}\right. \\
& \left.-\left(1+\beta^{2}\right)^{1 / 2}(\lambda \sin \theta)_{\theta} Y\right] d \theta
\end{aligned}
$$

where

$$
a=-\frac{1}{2 \pi}\left(1+\beta^{2}\right)^{1 / 2} J(X, Y) .
$$

In order to avoid the derivative of the function $\lambda$, which occurs in the right side of (4.10), we write, by partial integration, $\delta I(X, Y)$ in the form:

$$
\begin{aligned}
\delta I(X, Y)=-2 & \int_{0}^{2 \pi} \lambda\left[r\left(X_{r} X_{\theta}+Y_{r} Y_{\theta}\right)\right. \\
& \left.\quad-4 a\left(1+\beta^{2}\right)^{-1 / 2} r\left(X_{\theta} \cos \theta-\left(1+\beta^{2}\right)^{1 / 2} Y_{\theta} \sin \theta\right)\right] d \theta .
\end{aligned}
$$

Since $I(X, Y)=d$, the above expression should vanish for any continuously differentiable function $\lambda$ defined in the closed domain of the unit circle. Following Courant, we put

$$
\begin{aligned}
\lambda(u, v) & =\left(r^{2}-r_{0}^{2}\right) /\left(r^{2}+r_{0}^{2}-2 r r_{0} \cos \left(\theta-\theta_{0}\right)\right) \\
\text { (4.12) } \lambda(u, v) & =0 \text { for } 0<r \leqq 1, r_{0} \text { fixed, } r_{0}<\rho / 2, \\
\lambda(u, v) & \text { so defined in } \rho / 2<r \leqq \rho \text { that } \lambda \text { is continuously differentiable } \\
& \text { in } \bar{R} \text {. }
\end{aligned}
$$

Note that $\lambda(u, v)$ is the Poisson kernel for $r$ near $r=1$. With this $\lambda$ we consider beside (4.11) the following line integral $g(r)$

$$
g(r)=\frac{2 a}{\left(1+\beta^{2}\right)^{1 / 2}} \int_{0}^{2 \pi} \lambda\left(1-r^{2}\right)\left(X_{v}+\left(1+\beta^{2}\right)^{1 / 2} Y_{u}\right) d \theta
$$

and first prove

$$
\lim _{r \rightarrow 1} g(r)=0
$$


This is true because for $\rho<r<1$

$$
\begin{aligned}
g(r)= & 2 a\left(1+\beta^{2}\right)^{-1 / 2} \cdot\left(1-r^{2}\right) X_{v}\left(r_{0} \cos \theta_{0}, r_{0} \sin \theta_{0}\right) \\
& -2 a\left(1-r^{2}\right) Y_{u}\left(r_{0} \cos \theta_{0}, r_{0} \sin \theta_{0}\right)
\end{aligned}
$$

due to (4.12) and the fact that both $X_{v}$ and $Y_{u}$ are harmonic functions in the interior of the unit circle, and $r_{0}$ remains fixed as $r \rightarrow 1$. We form the expression

$$
\begin{aligned}
\delta I(X, Y)+\lim _{r \rightarrow 1} g(r) \\
=-\int_{r \rightarrow 1} \lambda\left[2 r\left(X_{r} X_{\theta}+Y_{r} Y_{\theta}\right)+4 a r\left(\frac{X_{\theta}}{\left(1+\beta^{2}\right)^{1 / 2}} \cos \theta-Y_{\theta} \sin \theta\right)\right. \\
+2 a\left(1-r^{2}\right)\left(X_{r} \sin \theta+r^{-1} X_{\theta} \cos \theta\right) /\left(1+\beta^{2}\right)^{1 / 2} \\
\left.+2 a\left(1-r^{2}\right)\left(Y_{r} \cos \theta-r^{-1} Y_{\theta} \sin \theta\right)\right] d \theta=0 .
\end{aligned}
$$

Comparing the integrand with (4.1) one finds the above equation is exactly

$$
\int_{0}^{2 \pi} \lambda \Im\left(w^{2} p(w)\right) d \theta=0 .
$$

But $w^{2} p(w)$ is a harmonic function, hence

$$
\int_{r \rightarrow 1}^{2 \pi} \lambda \Im\left(w^{2} p(w)\right) d \theta=r_{0}^{2} e^{2 i \theta_{0}} p\left(r_{0} e^{i \theta_{0}}\right)=0 .
$$

Since $r_{0} e^{i \theta_{0}}$ is an arbitrary point in the interior of the unit circle, it follows that $w^{2} p(w) \equiv 0$.

The real part of $w^{2} p(w)$ must now be a constant $\dot{c}$. If we put $w=0$ in the right side of (4.2), we find that

$$
c=2 a\left(1+\beta^{2}\right)^{-1 / 2}\left(X_{u}-\left(1+\beta^{2}\right)^{1 / 2} Y_{v}+2 a /\left(1+\beta^{2}\right)^{1 / 2}\right)_{w=0} .
$$

But according to (4.10) and the mean value theorem of harmonic functions we have

$$
\begin{aligned}
a & =-\left(1+\beta^{2}\right)^{1 / 2} J(X, Y) / 2 \pi \\
& =-\frac{1}{2 \pi}\left(1+\beta^{2}\right)^{1 / 2} \iint_{R}\left(X_{u}-\left(1+\beta^{2}\right)^{1 / 2} Y_{v}\right) d u d v \\
& =-2^{-1}\left(1+\beta^{2}\right)^{1 / 2}\left(X_{u}-\left(1+\beta^{2}\right)^{1 / 2} Y_{v}\right)_{w=0} .
\end{aligned}
$$

Hence $c=0$. Both the differential equations (4.1) and (4.2) are satisfied by the solution $X(u, v), Y(u, v)$ of the minimum problem.

THEOREM 2. By putting a solution of our minimum problem into (1.9) with $a=-\left(1+\beta^{2}\right)^{1 / 2} / 2 \pi \int_{0_{r-1}}^{2 \pi}\left(X \cos \theta-\left(1+\beta^{2}\right)^{1 / 2} Y \sin \theta\right) d \theta$ we obtain a minimal surface defined in $u^{2}+v^{2} \leqq 1$. The two functions $x(u, v), y(u, v)$ in (1.9) map 
the circle $u^{2}+v^{2}=1$ monotonically and continuously into $C$ and $\partial z / \partial r=0$ for $r=1$.

5. Continuous dependence of the solutions on $\beta$. Our integral $I$ depends on $\beta$ in a very simple form, since $\beta$ appears only in the line integral $J$. In order to express this dependence symbolically, we write, for any admissible pair of functions $f, g$, the integral in the form $I(f, g ; \beta)$. Let us also denote for any fixed $\beta$ the minimum of $I(f, g ; \beta)$ among all admissible pairs $f, g$, by $I(\beta)$. An admissible pair of functions $X, Y$ for which $I(X, Y ; \beta)$ attains the value $I(\beta)$ will be denoted by $X(u, v ; \beta), Y(u, v ; \beta)$ or simply $X(\beta), Y(\beta)$. Observe the fact that whatever constant $\beta$ we take in the minimum problem, the set of admissible functions is the same.

We should mention first of all the case $\beta=0$. From (1.9) we see that $z(u, v) \equiv 0$ for $\beta=0$. When we put a solution $X(u, v ; 0), Y(y, v ; 0)$ into (1.9), we get the identity $p(w) \equiv 0$ which means nothing else than

$$
\left(x_{u}-i x_{v}\right)^{2}+\left(y_{u}-i y_{v}\right)^{2} \equiv 0 .
$$

Thus either $x+i y$ or $x-i y$ is an analytic function of the complex variable $w$. Considering the orientation of the curve $C$ in which the unit circle $u^{2}+v^{2}=1$ is mapped, one finds $x+i y$ is analytic. Moreover the said mapping from the circle to $C$ must be topological, because it was required to be monotonic and continuous and because the analytic function $x+i y$ cannot be a constant along an arc of the circle. Following a standard argument it is seen that the mapping $w \rightarrow x+i y$ goes from $|w|<1$ into the exterior of $C$ and is one-to-one. Hence our function $x+i y$ gives us in this case the well known conformal mapping. If we restrict the constant $a$ in (1.9) to be positive, which can be done by eventually changing the coordinate system $(u, v)$ to $(-u,-v)$ without effecting the integral $I$, then the conformal mapping is uniquely determined. Therefore for $\beta=0$ the solution of the minimum problem $I(f, g ; 0)$ $=\min$ is unique. Furthermore the solution $X(u, v ; 0), Y(u, v ; 0)$ is differentiable on the boundary, if the curve $C$ is assumed to be smooth (a well known theorem which follows from theory of conformal mapping). When $\beta=0$, the normal direction of the surface at infinity is parallel to the $z$-axis. Thus it is geometrically evident that the solution should be the planes $z=$ const,

THEOREM 3. $I(\beta)$ is a continuous function of $\beta$.

Proof. For any two numbers $\beta_{1}$ and $\beta_{2}$ we have, due to the minimum property,

$$
\begin{aligned}
& I\left(\beta_{1}\right) \leqq I\left(X\left(\beta_{2}\right), Y\left(\beta_{2}\right) ; \beta_{1}\right), \\
& I\left(\beta_{2}\right) \leqq I\left(X\left(\beta_{1}\right), Y\left(\beta_{1}\right) ; \beta_{2}\right) .
\end{aligned}
$$

Hence, in the case $I\left(\beta_{1}\right) \leqq I\left(\beta_{2}\right)$ we get the inequality 


$$
\begin{aligned}
I\left(\beta_{2}\right)-I\left(\beta_{1}\right) & \leqq I\left(X\left(\beta_{1}\right), Y\left(\beta_{1}\right) ; \beta_{2}\right)-I\left(X\left(\beta_{1}\right), Y\left(\beta_{1}\right) ; \beta_{1}\right) \\
& =\frac{\left(\beta_{1}^{2}-\beta_{2}^{2}\right)}{\pi}\left(\int_{r=1} Y\left(\beta_{1}\right) d u\right)^{2} \\
& +2 \frac{\left(1+\beta_{1}^{2}\right)^{1 / 2}-\left(1+\beta_{2}^{2}\right)^{1 / 2}}{\pi} \int_{r=1} X\left(\beta_{1}\right) d v \int_{r=1} Y\left(\beta_{1}\right) d u
\end{aligned}
$$

and in case $I\left(\beta_{2}\right)<I\left(\beta_{1}\right)$ the same inequality as above with $\beta_{1}$ and $\beta_{2}$ interchanged. If we denote by $N$ an upper bound of the line integrals $\int_{r=1} f_{1} d v$ and $\int_{r=1} g d u$ for all admissible pairs $f, g$, then we have the estimate:

$$
\left|I\left(\beta_{1}\right)-I\left(\beta_{2}\right)\right| \leqq N^{2}\left(\frac{\left|\beta_{1}^{2}-\beta_{2}^{2}\right|}{\pi}+2 \frac{\left|\left(1+\beta_{1}^{2}\right)^{1 / 2}-\left(1+\beta_{2}^{2}\right)^{1 / 2}\right|}{\pi}\right) .
$$

Thus Theorem 3 is proved.

ThEOREM 4. From any sequence of solutions $X\left(\beta_{i}\right), Y\left(\beta_{i}\right), i=1,2,3 \cdots$, with $\beta_{i} \rightarrow \beta^{*}$, there is always a subsequence which converges to a solution $X\left(\beta^{*}\right)$, $Y\left(\beta^{*}\right)$.

Proof. Suppose it has been proved that the sequence $X\left(\beta_{i}\right), Y\left(\beta_{i}\right)$ is equicontinuous in $u^{2}+v^{2} \leqq 1$, then we can select a subsequence which converges to a limit pair, say, $f, g$. Since we have from Theorem 3

$$
I\left(\beta_{i}\right) \rightarrow I\left(\beta^{*}\right)
$$

and due to the lower semi-continuity of the Dirichlet integral and the continuity of the line integral $J$

$$
\begin{aligned}
D_{R}(f, g) & \leqq \lim \inf D_{R}\left(X\left(\beta_{i}\right), Y\left(\beta_{i}\right)\right), \\
J\left(f, g ; \beta^{*}\right) & =\lim _{\beta_{i} \rightarrow \beta^{*}} J\left(X\left(\beta_{i}\right), Y\left(\beta_{i}\right) ; \beta_{i}\right)
\end{aligned}
$$

it follows that $I\left(f, g ; \beta^{*}\right) \leqq I\left(\beta^{*}\right)$. Hence

$$
I\left(f, g ; \beta^{*}\right)=I\left(\beta^{*}\right)
$$

and $f, g$ is a solution $X\left(\beta^{*}\right), Y\left(\beta^{*}\right)$.

The equi-continuity of the sequence $X\left(\beta_{i}\right), Y\left(\beta_{i}\right)$ follows in the same way as in $\$ 3$.

Institute for Mathematics and Mechanics, New York University, NEW York, N. Y. 